\title{
A comparison of passive control methods for a cavity in transonic flow
}

\author{
A. J. Saddington, V. Thangamani ${ }^{\dagger}$ K. Knowles ${ }^{\ddagger}$ \\ Centre for Defence Engineering, Cranfield University, \\ Defence Academy of the United Kingdom, Shrivenham, Swindon, SN6 8LA, UK
}

\begin{abstract}
A comparative study of different passive control techniques was conducted on a cavity of length $320 \mathrm{~mm}$ with length-to-depth and length-to-width ratios of five and two respectively. The tests were conducted at a freestream Mach number of 0.71 . Both leadingedge and trailing-edge modifications were included in the studies. Results from surface pressure measurements showed that leading-edge control techniques were more effective at suppressing cavity tone amplitudes than trailing-edge modifications. A square-tooth spoiler showed the greatest reduction in tonal amplitude $(8.8 \mathrm{~dB})$, however, a sawtooth spoiler showed the greatest reduction in overall sound pressure level $(8.13 \mathrm{~dB})$. Velocity measurements inside the cavity were made using particle image velocimetry for the clean cavity and cavity with sawtooth spoilers. The results showed a reduction in momentum exchange between the freestream flow and the cavity when spoilers are used. This is proposed to be the main reason for the reduced tonal amplitudes.
\end{abstract}

\section{Nomenclature}

$C_{p} \quad$ pressure coefficient; $2 /\left(\gamma M^{2}\right) /\left(p / p_{\infty}-1\right)$.

$D \quad$ cavity depth [m].

$f \quad$ frequency $[\mathrm{Hz}]$.

$h \quad$ height of spoiler or other cavity feature (see Figure 5) [m].

$K$ ratio of disturbance velocity in the shear layer to the freestream velocity.

$L \quad$ cavity length $[\mathrm{m}]$.

$l \quad$ length of cavity feature (see Figure 5) [m].

*Senior Lecturer, Aeromechanical Systems Group.

${ }^{\dagger} \mathrm{PhD}$ Researcher

${ }^{\ddagger}$ Professor, Head of Aeromechanical Systems Group, AFAIAA. 


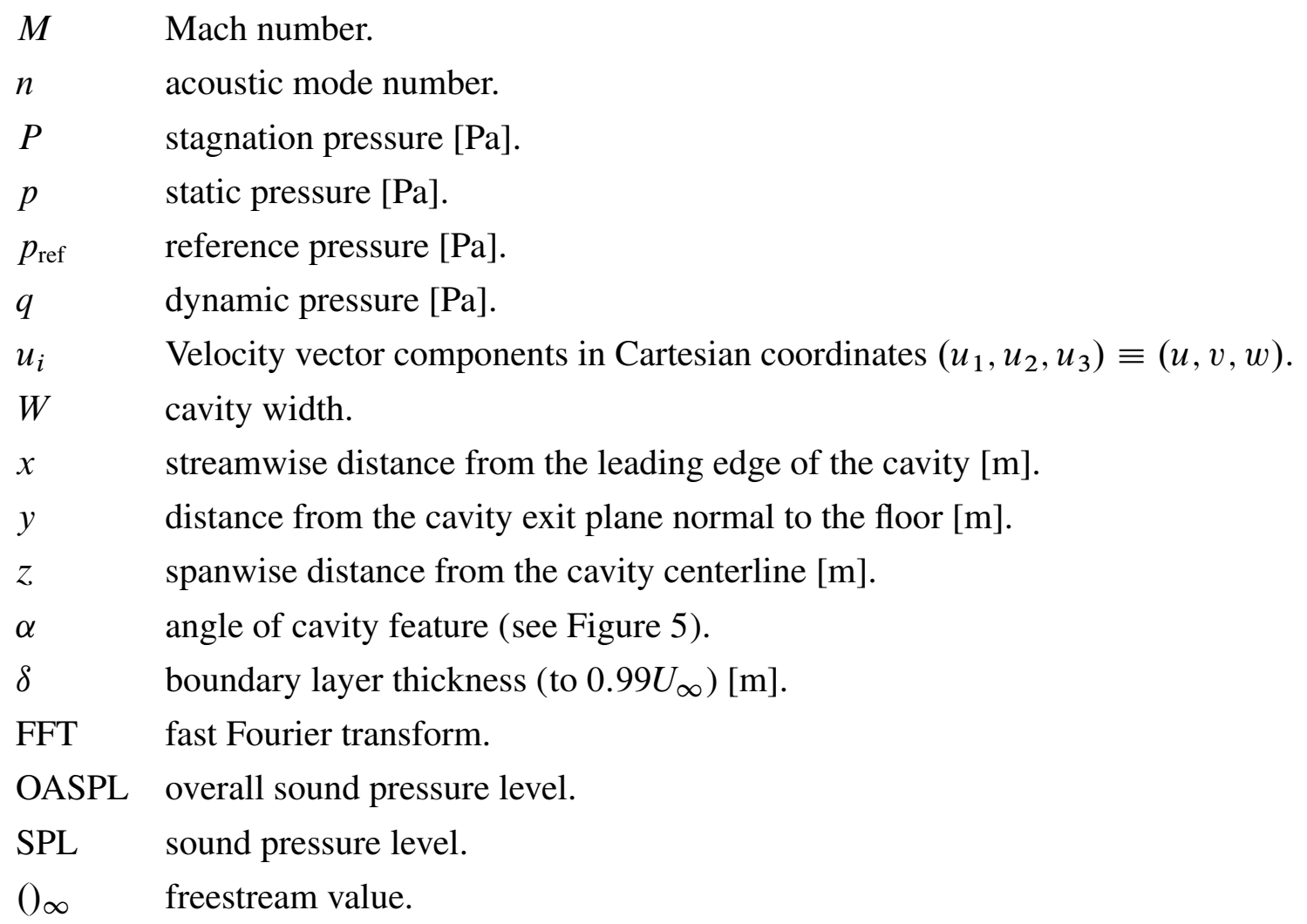

\section{Introduction}

M O DE R N fighter aircraft designs often employ internal weapons bays in order to reduce the radar signature of the aircraft in hostile environments, and for the additional advantage of drag reduction. Flow past the weapons bay when its doors are open can, however, lead to unfavourable conditions inside and around the bay. Cavity flows are usually classified by the lengthto-depth ratio, $L / D$, of the cavity. Following the experimental study of Stallings and Wilcox [1] four different types of cavity flow are found to be present depending on the value of $L / D$. The four flow types are: open; transitional-open; transitional-closed; closed. Open cavity flows are generally prone to unsteady pressure tones, which can sometimes have amplitudes high enough to damage the aircraft components. Closed cavity flows do not exhibit pressure tones, however, they do create a stream-wise adverse pressure gradient on the cavity ceiling, which can cause a nose-in pitching moment on the store and interfere with its accurate release. Transitional cavity flows show a combination of open and closed cavity flow phenomena.

The characteristic frequencies associated with cavity oscillations are first predicted by Rossiter [2] but now are generally predicted using the modified Rossiter equation proposed by Heller et al. [3], which accounts for the higher speed of sound within the cavity at high freestream Mach num- 
bers.

$$
f_{n}=\frac{U_{\infty}}{L}\left(\frac{n-\alpha}{M_{\infty}\left[1+\frac{(\gamma-1)}{2} M_{\infty}^{2}\right]^{-\frac{1}{2}}+\frac{1}{K}}\right)
$$

where $f_{n}$ is the frequency corresponding to the $n_{\text {th }}$ mode, $K$ is the ratio of disturbance velocity in the shear layer to the freestream velocity (generally taken as 0.57 ) and $\alpha$ is an empirical constant employed to account for the phase lag between the passage of a vortical disturbance past the cavity trailing edge and the formation of an upstream travelling disturbance. The value of $\alpha$ depends on $L / D$ and is evaluated as : $\alpha=0.062(L / D)$. The ratio of specific heats, $\gamma$, is assumed to be equal to 1.4 for air.

The control of cavity flow oscillations is of practical significance and has, consequently, been the subject of numerous studies [4-12]. The flow control method can be passive or active depending on the external energy input [13]. Active control has the advantage that closed-loop feedback can be included to adapt to different frequencies. On the other hand passive control methods involve simple geometric modifications and do not involve any external energy input. They are generally cheaper than active control methods and attenuate the cavity oscillation by disrupting the Rossiter feedback mechanism [2] which is responsible for the oscillations. Previous research has showed that the geometrical scale of the cavity has a decisive influence on the noise levels and flow type of a cavity [14]. The noise levels and acoustic tone amplitudes were found to increase with $L / \delta$ (where $\delta$ is the onset boundary layer thickness) and the control of cavity tones becomes more difficult at higher values of $L / \delta$. It was also seen that the flow type changed from open to transitional when $L / \delta$ was increased from 10 to 41 This entailed an increase in time-averaged pressure gradients formed along the cavity length. As a result of these observations it is of practical importance to test the control techniques on as large a scale as possible. This includes both leading-edge and trailing-edge modifications.

In this current work, 13 different passive control techniques have been studied on a $320 \mathrm{~mm}$ long cavity model to compare their effectiveness in the suppression of cavity tones and their effect on the pressure gradients established on the cavity floor. Unsteady pressure measurements have been taken on the cavity floor (ceiling) to study the acoustic signature and noise generated in the clean cavities as well as in cavities with passive control. The suitability of different passive control techniques for preventing the unfavourable pressure gradients inside the cavity has also been studied.

\section{Experimental details}

This section details the wind tunnel facility and test rig, including a description of the models and the different passive control techniques that have been used in the experiments. The experiments were conducted in a transonic wind tunnel with the cavity models attached to the side wall of the 
test section.

\section{A. Transonic wind tunnel}

The experimental studies were carried out in the closed-circuit, ejector-driven transonic wind tunnel facility at the Defence Academy of the United Kingdom, Shrivenham. A general layout of the wind tunnel is shown in Figure 1. The wind tunnel has a working section that is $500 \mathrm{~mm}$ long, $206 \mathrm{~mm}$ high and $228 \mathrm{~mm}$ wide. Using a Compair L110-10, air is compressed, dried and stored at $724 \mathrm{kPa}$ (gauge) in a $34 \mathrm{~m}^{3}$ reservoir that supplies the air to the wind tunnel. The tunnel has a run time of approximately $8 \mathrm{~s}$ for a computer-controlled blowing pressure of approximately $500 \mathrm{kPa}$ (gauge) at a tunnel stagnation pressure of $102.22 \mathrm{kPa}$. For the experiments presented here the tunnel working section Mach number was set to 0.71 giving a mean static pressure of $73.07 \mathrm{kPa}$. The uncertainty in the centre-line Mach number was obtained from five tunnel runs of 65536 samples each and calculated at $95 \%$ confidence level as \pm 0.01 . The Reynolds number per unit length was calculated to be $13.1 \times 10^{6} \mathrm{~m}^{-1}$.

The natural boundary layer developed over the tunnel wall was used for the studies. The onset boundary layer thickness was estimated by measuring the stagnation pressure profile (see Figure 2 ) at a distance $45 \mathrm{~mm}(0.14 \mathrm{~L})$ upstream of the leading edge of the cavity model. The boundary layer thickness, $\delta$, was defined as the height from the wall to where $u / u_{\infty}=0.99$. This value was determined from the measured profile to be $8.0 \mathrm{~mm}$.

\section{B. Cavity rig}

The cavity was rectangular with dimensions of $L \times W \times D=320 \mathrm{~mm} \times 160 \mathrm{~mm} \times 64 \mathrm{~mm}$, giving $L / D$ and $L / W$ as 5 and 2 respectively. The cavity rig was attached to the side wall of the wind tunnel test section (see Figure 1). The reference axis system used for the experiments is shown in Figure 3; the origin is at the centre of the leading edge of the cavity. The $x$-axis is parallel to the flow direction, while the $y$-axis is normal to the tunnel wall. Nine pressure tappings of $0.8 \mathrm{~mm}$ diameter were spaced linearly in the streamwise direction at increments of $x / L=0.1$. Three such rows are placed at planes $z / W=0, z / W=0.1625$ and $z / W=0.325$ which are referred to as "Centreplane" (CP), "Offsetplane1" (OP1) and "Offsetplane2" (OP2) respectively. In total, there were 27 static pressure tappings for the cavity model, forming a measurement grid on the floor as shown in Figure 4. Flow visualization of a cavity with the same $L / D$ and $L / W$ ratios by Ritchie [15] indicated that the cavity flow-field is reasonably symmetric about the centreline. Hence symmetry is assumed and the pressure tappings have been made only for half of the floor plane. 


\section{Passive control devices}

Thirteen different passive control techniques were tested and the following section gives details of the different control methods. Only those passive control methods which did not alter the cavity floor area were selected for this study. This enabled a consistent comparison of the pressure data obtained from the measurement grid on the cavity floor.

Leading-edge spoilers with three different profiles were tested: flat top; saw tooth; and square tooth (Figures 5a - 5c). The sawtooth spoilers are composed of triangular elements. The blockage ratio of all the sawtooth and square-tooth spoilers is maintained equal for consistency. Rossiter [2] reported maximum tone suppression when the spoiler height equalled the boundary layer thickness. Hence, the height of all the spoilers was chosen to be $8 \mathrm{~mm}$, which is equal to the incoming boundary layer thickness in the current study. In addition to the spoilers, a leading-edge wedge (Figure 5d) of the same height was also tested. The spoilers were securely attached to the front wall of the cavity to prevent vibrations during the tests. All the spoilers were made of $2 \mathrm{~mm}$-thick aluminium sheet and a pocket of $2 \mathrm{~mm}$ was given in to the front wall of the cavity in order to maintain the same $L / D$.

Porosity of the cavity walls could have an effect on the cavity oscillations. A porosity of $50 \%$ was used on the aft and front walls (Figures 5e and 5f). The hypothesis behind the effect of porous walls is that porous walls can diffuse and dissipate the pressure waves generated, which leads to weakening of the acoustic resonant feedback. Ramps have been found to be effective in suppression of cavity oscillations in the studies of Kok et al. [16] and Vikramaditya and Kurian [12]. Only trailing-edge ramps were used in these previous studies; in the present study, however, the ramp was tested both at the leading and trailing edges (Figures $5 \mathrm{~g}$ and $5 \mathrm{~h}$ ) to enable a direct comparison between the two configurations. Vikramaditya and Kurian [12] also noted that the suppression due to ramps and positively-angled slant walls gave similar levels of suppression. Negatively-angled slant walls (Figures 5i and 5j) were, however, not tested and it was deemed useful in the present study to determine their effect on the unsteady pressure tones.

Thickening the boundary layer has been found to reduce the amplitude of cavity oscillations [17]. Based on this concept, a leading-edge step (Figure 5k), which is postulated to thicken the boundary layer and thus reduce the cavity unsteadiness, was tested. The leading-edge step acts like a backward-facing step and thus thickens the boundary layer due to separation. The height of the step was chosen as $10 \mathrm{~mm}$ which is close to the boundary layer thickness used in this study. The reattachment of the shear layer for a backward facing step takes places at a downstream distance of approximately $60 \%$ to $90 \%$ of the step height [18]. Sufficient length has been given in the leadingedge step used in this study (100\% of step height) to ensure reattachment on the step floor. The step was also placed at the trailing edge (Figure 51) to test if it has an effect on cavity unsteadiness by influencing the interaction between the shear layer and the trailing edge. An alternative approach to thicken the boundary layer, a deep cavity placed near the leading edge, was also tested (Figure 
$5 \mathrm{~m})$. This is postulated to thicken the boundary layer by perturbing it in the wall-normal direction thus contributing to cavity oscillation control. The $L / D$ of the leading-edge deep cavity used in this study was 0.4 . The specifications of the different passive control devices are given in Table 1 .

\begin{tabular}{lll}
\hline Passive control method & Abbreviation & Parameters (see Figures 5a - 5m) \\
\hline Flattop spoiler & FTS & $h_{\mathrm{FTS}} / \delta=1$ \\
Sawtooth tooth spoiler & SWS & $h_{\mathrm{SWS}} / \delta=1$ \\
Square-tooth spoiler & $\mathrm{STS}$ & $h_{\mathrm{STS}} / \delta=1$ \\
Leading-edge wedge & $\mathrm{LW}$ & $h_{\mathrm{LW}} / \delta=1, \alpha_{\mathrm{LW}}=38.65^{\circ}$ \\
Porous front wall & $\mathrm{PFW}$ & $l_{\mathrm{PFW}} / L=0.0625$, Porosity $=50 \%$ \\
Porous aft wall & $\mathrm{PAW}$ & $l_{\mathrm{PAW}} / L=0.0625$, Porosity $=50 \%$ \\
Leading-edge ramp & $\mathrm{LR}$ & $h_{\mathrm{LR}} / D=0.35, \alpha_{\mathrm{LR}}=46^{\circ}$ \\
Trailing-edge ramp & $\mathrm{TR}$ & $h_{\mathrm{TR}} / D=0.35, \alpha_{\mathrm{TR}}=46^{\circ}$ \\
Aft-wall slant & $\mathrm{AWS}$ & $l_{\mathrm{AWS}} / L=0.19, \alpha_{\mathrm{AWS}}=44^{\circ}$ \\
Front-wall slant & $\mathrm{FWS}$ & $l_{\mathrm{FWS}} / L=0.19, \alpha_{\mathrm{FWS}}=44^{\circ}$ \\
Leading-edge step & $\mathrm{LS}$ & $h_{\mathrm{LS}} / \delta=1.25, l_{\mathrm{LS}} / \delta=1.25$ \\
Trailing-edge step & $\mathrm{TS}$ & $h_{\mathrm{TS}} / \delta=1.25, l_{\mathrm{TS}} / \delta=1.25$ \\
Leading-edge deep cavity & $\mathrm{LDC}$ & $h_{\mathrm{LDC}} / \delta=2.125, l_{\mathrm{LDC}} / \delta=0.875$ \\
\hline
\end{tabular}

Table 1: Summary of the passive control methods used.

\section{Instrumentation}

\section{Pressure measurements}

The unsteady pressure measurements in the experimental tests were made with an integrated pressure measurement device, the Scanivalve ZOC22B and data were collected from the pressure tappings made on the cavity floor. The analogue signal from the ZOC22B was acquired by a modular amplifier system (DEWE-RACK) which has an analogue-to-digital converter. The signal was amplified, conditioned and digitized before being sent to a computer. A National Instruments DAQ card 6036E was used as the interface between the computer and the DEWE-RACK. For the spectral analysis of the unsteady pressure samples, a total of 65536 samples, averaged over four tunnel runs, were recorded at a sampling rate of $12.5 \mathrm{kHz}$. The power spectra were plotted using an FFT algorithm to determine the amplitude of the different frequency components. The maximum error in amplitude of the tones was determined to be $0.208 \mathrm{~dB}$. The same time series was used in constructing the spectrograms. 


\section{Velocity measurements}

Two-dimensional velocity field measurements were made using particle image velocimetry (PIV) inside a purpose-built, transparent cavity. The PIV equipment consisted of a New Wave Gemini II Nd:YAG double-pulsed laser which, through the use of a pair of spherical lenses, created a light sheet approximately $1 \mathrm{~mm}$ thick. Since the wind tunnel had no optical access at right angles to the laser sheet, the image was obtained using a front-coated mirror oriented at 45 degrees to the cavity side wall (Figure 6). With the exception of the transparent floor and wall, all the sides of the cavity models and the test rig were painted black for the PIV experiments to prevent stray reflections of the laser.

Water was used for seeding the flow. Filtered clean water was pressurised using a three-stage pump and sprayed through a rake of BETE ${ }^{\circledR}$ atomizing nozzles positioned across the span of the settling chamber of the wind tunnel. The rake consisted of three atomizers located at equal intervals at the mid-height position of the test section. The nominal seeding particle diameter based on a seeding pressure of 2000 psi was estimated to be $10 \mu \mathrm{m}$.

The PIV double-pulsed image pairs were acquired using a Kodak Megaplus ES1.0 digital camera with a resolution of $1016 \times 1008$ pixels at a rate of 15 image pairs per second and a pulse separation of $6 \mu \mathrm{s}$. A Dantec Flow Map 500 acquisition controller was used as the hardware box, which worked in conjunction with Flow Manager (v3.21) software. Both the camera and laser were connected to the hardware box and the timing and acquisition functions of both were controlled using the Flow Manager software. A window deformation algorithm, with initial and final interrogation sizes of $32 \times 32$ pixels and $12 \times 12$ pixels respectively, was used for post-processing the image data. All the measurements presented in this work were obtained from the mid plane of the cavity $(z / W=0)$. The time-averaged data were obtained from the ensemble average of 700 image pairs. The maximum error in velocity measurement was estimated as $\pm 2.5 \%$.

\section{Results and discussion}

Figures 7 - 12 show the effect of the different passive control devices on the power spectrum as compared against that of the clean cavity $(\mathrm{CC})$. The frequency peaks for the clean cavity correspond to the Rossiter modes and are known as Rossiter tones. The frequencies of the Rossiter tones are invariant with the change in cavity measurement location and show the global oscillation nature. For convenience, oscillation modes 1, 2 and 3 of the frequency spectrum will be referred to as R1, R2 and R3 respectively in this work, and have been calculated using Equation 1.

It can be seen that there is a significant variation in the suppression of the tones by the different methods. Leading-edge spoilers show the maximum attenuation of cavity tones and noise near the trailing edge (Figure 7). Of these spoilers, the square-tooth spoiler (STS) shows maximum suppression of the tones with R2 (the dominant mode for the clean cavity) being reduced to $153.4 \mathrm{~dB}$, 
which is a reduction of $8.8 \mathrm{~dB}$. This is a large change and is followed by the flat top spoiler, FTS $(8.5 \mathrm{~dB})$, the leading-edge wedge, $\mathrm{LW}(7.5 \mathrm{~dB})$ and the sawtooth spoiler, SWS $(7.4 \mathrm{~dB})$ respectively. Another observation from Figure 7 is the increase in the tonal frequencies when the leading-edge spoilers are used. This increase in frequency may be caused by an increase in convective velocities of vortices in the deflected shear layer and/or an increase in the reverse velocities inside the cavity with the use of spoilers. Similar increases in frequency with the use of leading-edge spoilers was observed by Lawson and Barakos [5]. It should be noted that even with the presence of control devices, the tones are not completely attenuated.

Certain passive controls methods involved modifications to the walls of the cavity and these methods were tested on both the front and aft walls. Such testing revealed interesting variations in the suppression results. While the front-wall slant (FWS) reduced R 2 by only $1.2 \mathrm{~dB}$ and increased R1 by $0.8 \mathrm{~dB}$, it completely attenuated R3 to a broadband noise level (Figure 8 ). The aft-wall slant (AWS), by contrast had no effect on tonal suppression and, in fact, both increased the amplitude of the tones and introduced additional frequency modes. Whilst the trailing-edge ramp (TR) decreased the tone intensity slightly, the leading-edge ramp (LR) increased it by a similar amount (Figure 9). The remaining methods tested (LDC, PAW, PFW, LS and TS) were not found to be very effective in tone suppression as can be seen from Figures 10 to 12.

The overall sound pressure level (OASPL) reduction was calculated along the cavity for the different passive control devices (Figure 13) length and showed variations at different measurement points. The leading-edge spoilers FTS, SWS, LW and STS showed a significant reduction in OASPL throughout the cavity. The maximum reduction is $11.05 \mathrm{~dB}$ for STS at $x / L=0.4$. The square-tooth spoiler (STS) shows the maximum average reduction in OASPL across different locations $(8.13 \mathrm{~dB})$. This is followed by the sawtooth spoiler with an average reduction of $7.9 \mathrm{~dB}$ and then by the flat-top spoiler (FTS) and leading-edge wedge (LW) which have the same average reduction values $(7.65 \mathrm{~dB})$. From previous studies, it is known that the height of the spoilers plays a crucial role in noise suppression [2,9]. However, the results here show that the profile of the leading-edge spoiler also plays a significant role in noise suppression. The variation of the OASPL reduction along the cavity for FTS, SWS, LW and STS is similar to the mode shape of the dominant tone $\mathrm{R} 2$ of the clean cavity. This shows that the reduction in noise has resulted significantly from the reduction of the amplitude of the dominant tone. Thus, these methods have a direct impact in disrupting the Rossiter mechanism of cavity oscillation and thus reducing the tonal amplitudes. The shapes of the OASPL-reduction curves for the aft-wall slant (AWS) and the front-wall slant (FWS) differ from these curves because these methods do not have a significant effect on R2, as was seen in their unsteady power spectra (Figure 8). The OASPL reductions for FWS result from the suppression of R3 and R2 as well as a decrease in the broadband noise. The other passive control methods do not show a significant noise reduction and at some points certain methods, like the leading-edge step (LS) and leading-edge ramp (LR), increase the amplitude. 
As mentioned in the introduction, the steep pressure gradient established on the cavity floor for some cavity geometries can be unfavourable for store release and it is therefore pertinent to reduce the gradients established along with the suppression of flow unsteadiness. Figure 14 shows the time-averaged pressure on the floor along the cavity length. It can be seen that there is a steep pressure gradient for the clean cavity (CC), which is typical of a transitional cavity flow. Most of the passive control devices reduce the pressure inside the cavity. The leading-edge spoilers, which were shown to have very good tone suppression results, can be seen to reduce the cavity floor pressures significantly. In addition to the reduction, the pressure is also approximately uniform for up to $80 \%$ of the cavity length. The pressure magnitudes increases slightly towards the trailing edge indicating a very weak interaction there between the cavity and flow. For the leading-edge wedge (LW) and the flat top spoiler (FTS), the absolute pressure is reduced by an average of $23 \%$ up to $x / L=0.7$, while for the square-tooth and sawtooth spoilers (STS and SWS) the value is $19 \%$. This suggests that the pressure reduction inside the cavity is a result of the flow being deflected away from the cavity. For the leading-edge wedge and flattop spoiler (LW and FTS), the blockage to the flow is high and results in more deflection of the flow away from the cavity and lower pressure inside the cavity. The front-wall slant (FWS) also shows a reduction in absolute pressure (by $7 \%$ ). The gradient established in this case is smoother than with the spoilers and is slightly more favourable for the release of stores. This can have practical implications since the front-wall slant (FWS) produces less drag compared to the leading-edge spoilers and the angle $\left(\alpha_{\mathrm{FWS}}\right)$ can be optimised to disrupt the pressure gradients inside and ensure the safe release of stores. The other passive control methods show only a slight flattening of the pressure gradients and are not as significant.

Based upon observations of the frequency spectra it can be concluded that better suppression levels are observed for cavities fitted with leading-edge passive control mechanisms. The velocity field inside the cavity gives insight to the possible mechanisms responsible for the suppression of tones observed in the power spectra. To assess this, PIV was used to measure the mean flow field of the clean cavity and the cavity with a selected leading-edge control; the sawtooth spoiler (SWS) was chosen. There is a primary recirculation region occupying up to the first $60 \%$ of the cavity length and a secondary recirculation region near the aft wall (Figure 15). The secondary recirculation region is caused by a dipping of the shear layer into the cavity. Figure 15 also shows that with the use of spoilers the flow inside the cavity changes significantly. The twin-recirculation zones are replaced by a single and larger recirculation region, centred further downstream. This recirculation region is less susceptible to unsteadiness and is a consequence of the change in flow stability within the cavity caused by the sawtooth spoilers.

For a given cavity, the streamwise component of the mean velocity gives an indication of the mean momentum flux transferred to the aft wall and consequently the strength of the acoustic disturbance and the feedback cycle. For the clean cavity, it can be observed that the region (inside the cavity) of high velocity flux in the streamwise direction is large (Figure 16a). This indicates 
a high momentum transfer from the freestream into the cavity, which leads to the high amplitude tones and noise observed in the power spectra. With the use of spoilers, however, it can be seen from Figure $16 \mathrm{~b}$ that the size of the region of high streamwise velocity flux has decreased. This results in a reduction in the strength of the feedback loop. The maximum streamwise velocity in the measurement area for the clean cavity is $96.4 \mathrm{~ms}^{-1}$; with the use of the sawtooth spoilers it reduces

to $65.3 \mathrm{~ms}^{-1}$. The reduction in the peak velocity is due to the deflection of the flow away from the cavity by the spoilers. This can be observed from the contours of vertical velocity (Figure 17). The region in the plane which has high negative values of $v$ indicates the mass entering into the cavity. For the clean cavity, the mass flow enters from $x / L=0.3$ of the cavity length (Figure 17a), however, with the use of the sawtooth spoilers, mass flow entering the cavity is shifted downstream to $x / L=0.6$ (Figure $16 \mathrm{~b}$ ).

\section{Conclusions}

A comparative study of 13 different passive control techniques on a $320 \mathrm{~mm}$ long cavity was conducted. Power spectral studies reveal that the leading-edge spoilers (square-tooth spoiler (STS), sawtooth spoiler (SWS), flattop spoiler (FTS) and leading-edge wedge (LW)) were more effective in suppressing the Rossiter tones than modifications to the cavity walls or the trailing edge. They also show maximum effectiveness in the reduction of OASPL across the cavity length. The suppression of the dominant tone near the aft wall was highest for STS ( $8.8 \mathrm{~dB})$ followed by FTS $(8.5 \mathrm{~dB})$, LW $(7.5 \mathrm{~dB})$ and SWS $(7.4 \mathrm{~dB})$. The performance of the leading-edge spoilers were better in terms of OASPL reduction and reducing the mean pressure gradient on the floor. STS and SWS showed the highest average reduction of OASPL along the centreline of $8.13 \mathrm{~dB}$ and $7.9 \mathrm{~dB}$ respectively. The reduction in noise obtained inside the cavity is deduced to be a consequence of the shear layer being deflected away from it by the leading-edge spoiler. Front-wall slant (FWS) also appears to be a promising passive control technique because it achieves an overall satisfactory performance in tonal amplitude suppression, noise control and favourable pressure gradient inside the cavity with no protrusion into the freestream flow. The other methods tested achieved lower suppression results compared to the above. Two-dimensional velocity measurements were made at the mid plane of the clean cavity and the cavity with sawtooth spoiler. The velocity field of the two cases revealed a change in the flow inside the cavity, and also indicated a reduction in the energy exchange between the cavity and the freestream, with the use of spoilers. This reduction in the energy exchange is believed to beresponsible for the reduction in tone amplitudes observed in the pressure spectra. 


\section{References}

1 R. Stallings and F. Wilcox. Experimental cavity pressure distributions at supersonic speeds. Tech. Rep. TP-2683, NASA, Langley Research Center; Hampton, VA, June 1987.

2 J. E. Rossiter. Wind-tunnel experiments on the flow over rectangular cavities at subsonic and transonic speeds. Reports and Memoranda 3438, Aeronautical Research Council, 1966.

3 H. Heller, D. Holmes, and E. Covert. Flow-induced pressure oscillations in shallow cavities. $J$. Sound Vib., 18:545-553, 1971.

4 C. Lada and K. Kontis. Experimental studies of open cavity configurations at transonic speeds with flow control. J. Aircraft, 48(2):719-724, 2011.

5 S. J. Lawson and G. N. Barakos. Assessment of passive flow control for transonic cavity flow using detached-eddy simulation. J. Aircraft, 46(3):1009-1029, 2009.

6 D. G. MacManus and D. S. Doran. Passive control of transonic cavity flow. J. Fluid Eng.-T ASME, 130(6):064501-1-064501-4, 2008.

7 M. A. Martinez, G. M. Di Cicca, M. Iovieno, and M. Onorato. Control of cavity flow oscillations by high frequency forcing. J. Fluid Eng.-T ASME, 134(5):051201-1-051201-11, 2012.

8 S. W. Perng and D. S. Dolling. Suppression of pressure oscillations in high-Mach-number, turbulent, cavity flow. J. Aircraft, 38(2):248-256, 2001.

9 L. Shaw, R. Clark, and D. Talmadge. F-111 generic weapons bay acoustic environment. $J$. Aircraft, 25(2):147-153, 1988.

10 L. S. Ukeiley, M. K. Ponton, J. M. Seiner, and B. Jansen. Suppression of pressure loads in cavity flows. AIAA J., 42(1):70-79, 2004.

11 N. S. Vikramaditya and J. Kurian. Effect of aft wall slope on cavity pressure oscillations in supersonic flows. Aeronaut. J., 113(1143):291-300, 2009.

12 N. S. Vikramaditya and J. Kurian. Pressure oscillations from cavities with ramp. J. Aircraft, 47 (12):2974-2984, 2009.

13 L. N. Cattafesta, Q. Song, D. R. Williams, C. W. Rowley, and F. S. Alvi. Active control of flow-induced cavity oscillations. Prog. Aerosp. Sci., 44(7-8):479-502, 2008.

14 V. Thangamani, K. Knowles, and A. J. Saddington. The effects of scaling on high subsonic cavity flow oscillations and control. J. Aircraft, 51(2):424-433, 2014. 
15 S. A. Ritchie. Non-intrusive measurements and computations of transonic cavity flows with applications to aircraft stores release. $\mathrm{PhD}$ thesis, Cranfield University, 2005.

16 J. C. Kok, B. I. Soemarwoto, and H. Ven. X-LES simulations using a high-order finite-volume scheme. Note Num. Fl., 97:87-96, 2008.

17 N. Zhuang, F. S. Alvi, M. B. Alkislar, and C. Shih. Supersonic cavity flows and their control. AIAA J., 44(9):2118-2128, 2006.

18 E. B. Plentovich and R. L. Stallings M. B. Tracy. Experimental cavity pressure measurements at subsonic and transonic speeds. Tech. Rep. TP-3358, NASA, Langley Research Center, Hampton, VA, 1993.

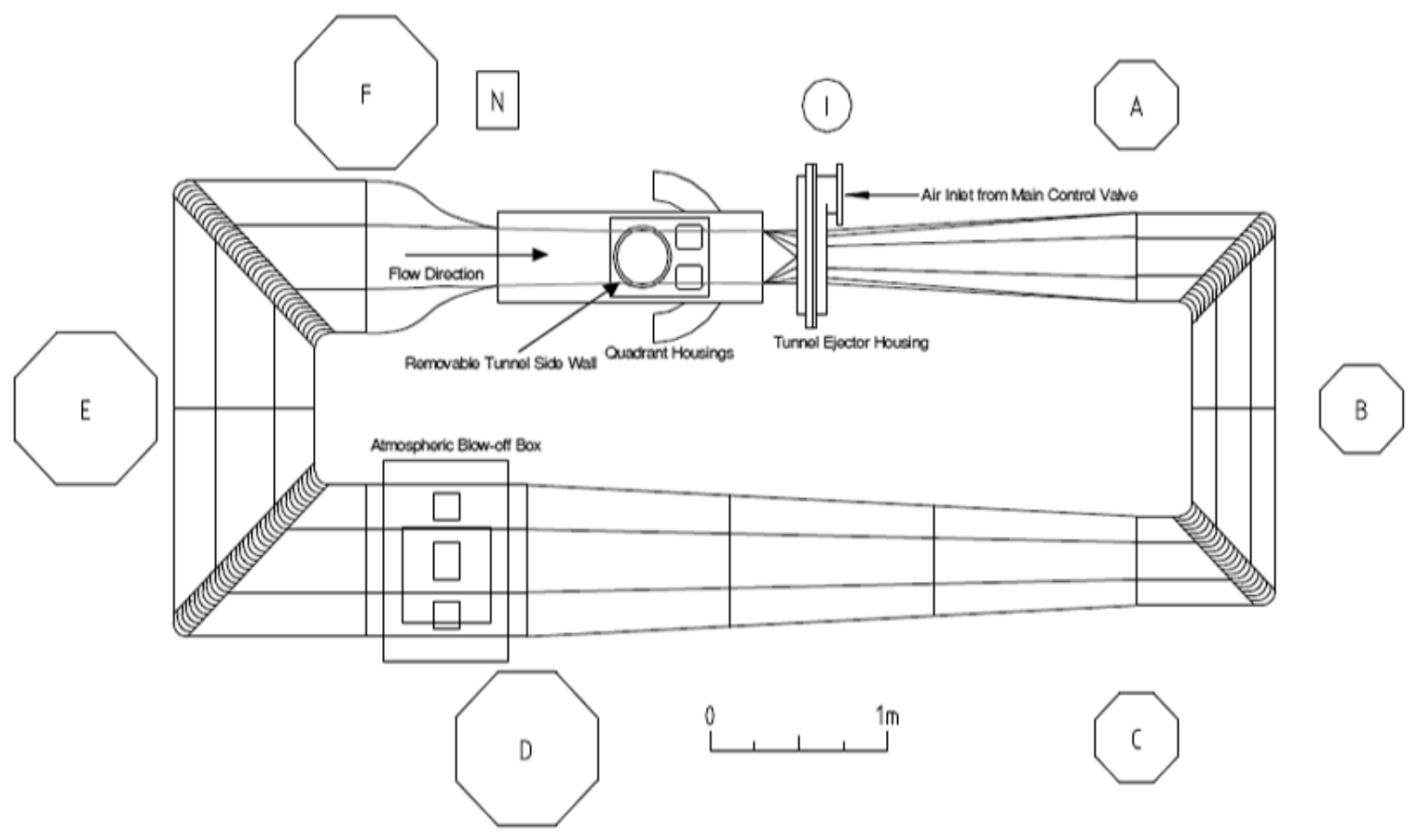

Figure 1: Transonic wind tunnel layout - Sections A-F show wind tunnel cross sections at various points ; $\mathrm{N}$ and I are the nozzle and ejector planes respectively. 


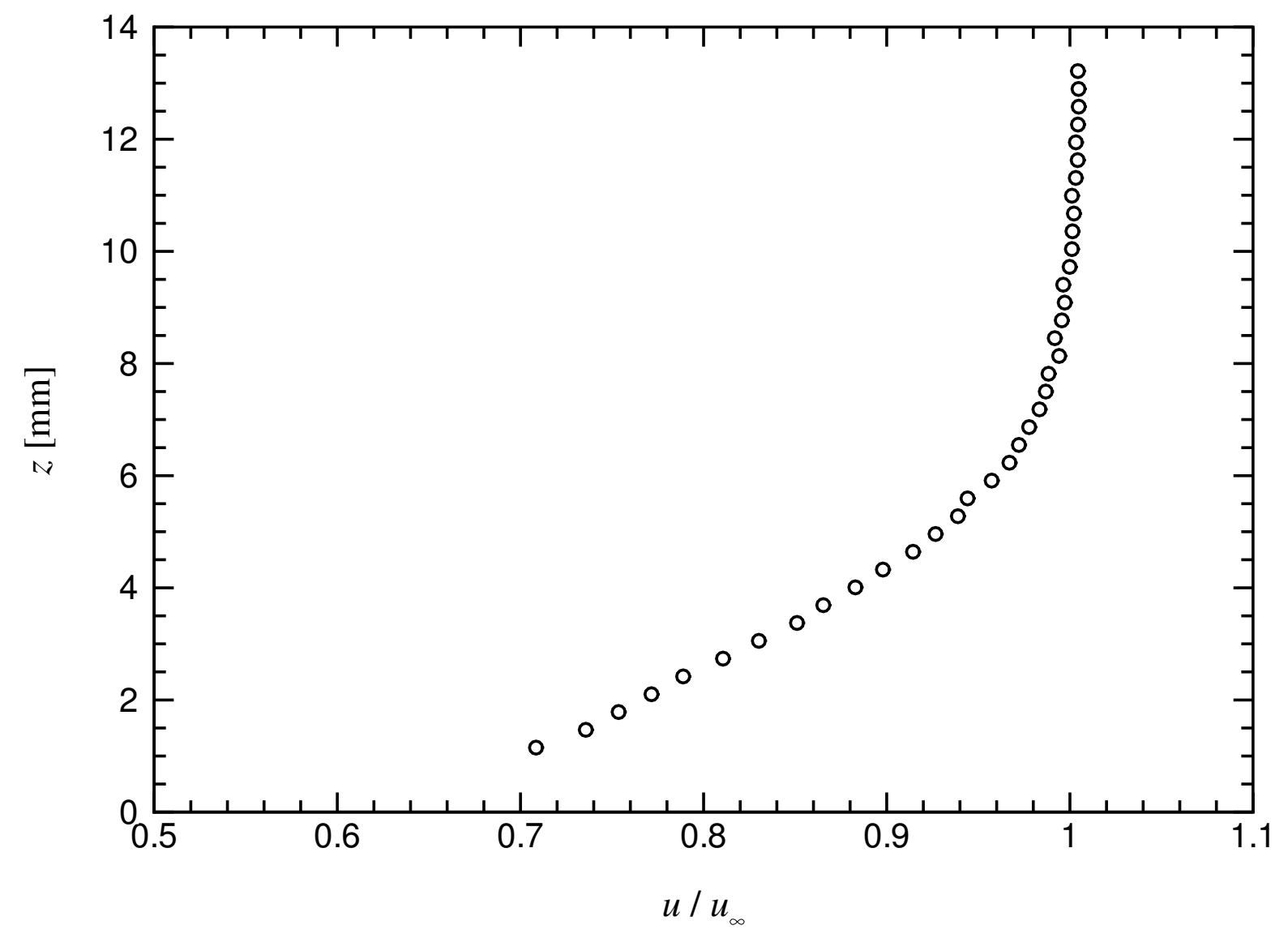

Figure 2: Boundary layer profile $0.14 L$ upstream of the cavity leading edge. 


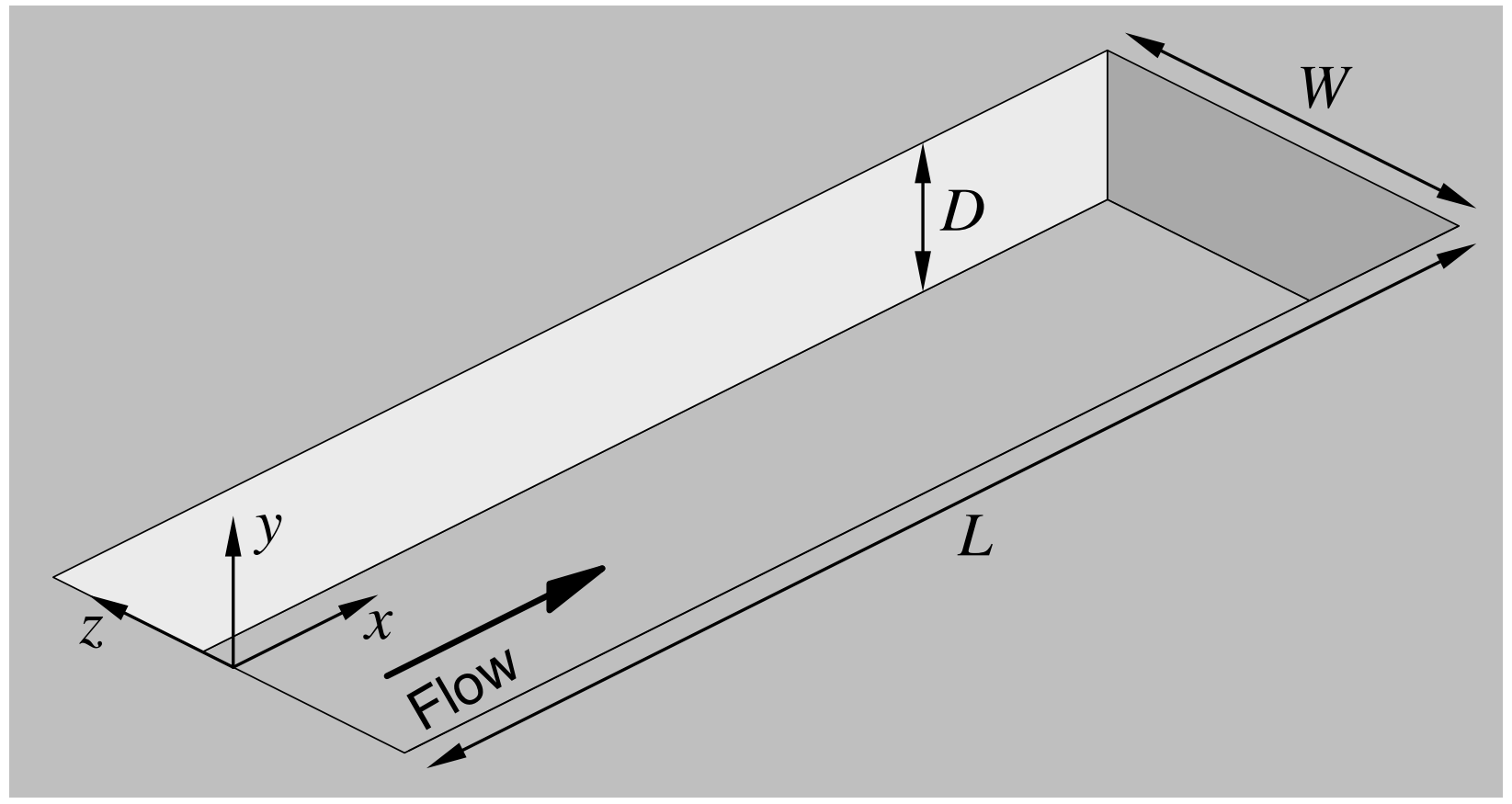

Figure 3: Cavity coordinate system.

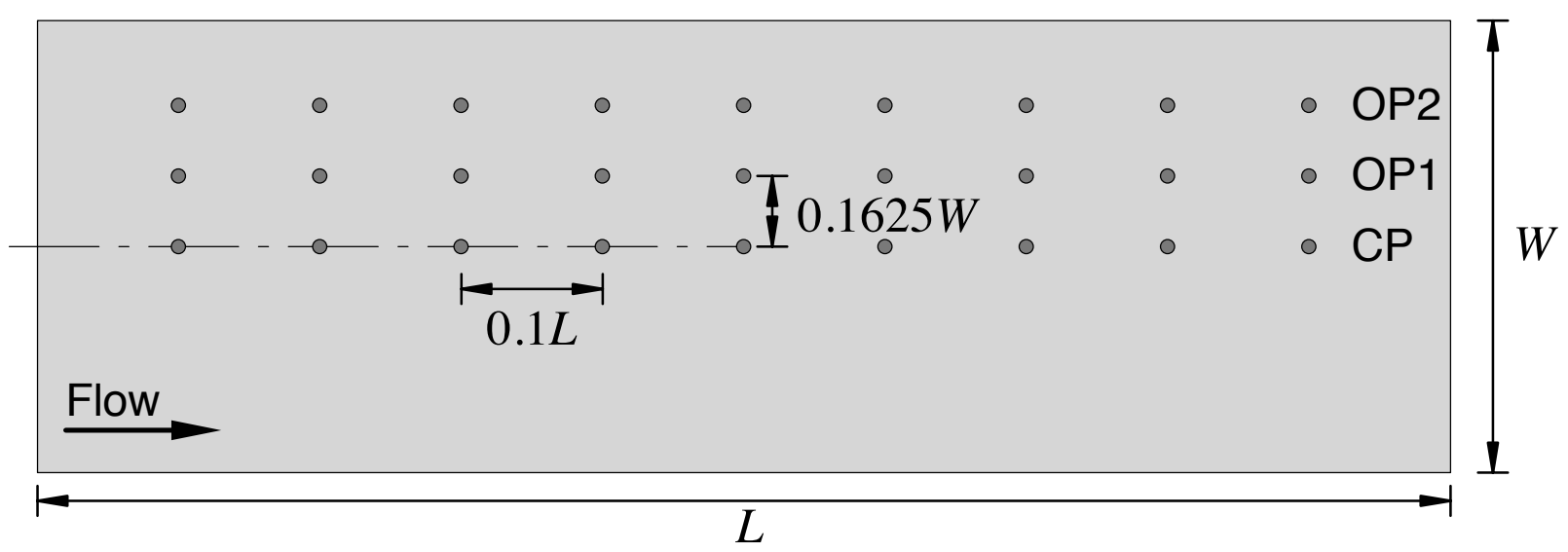

Figure 4: The arrangement of pressure tappings on the cavity floor. 


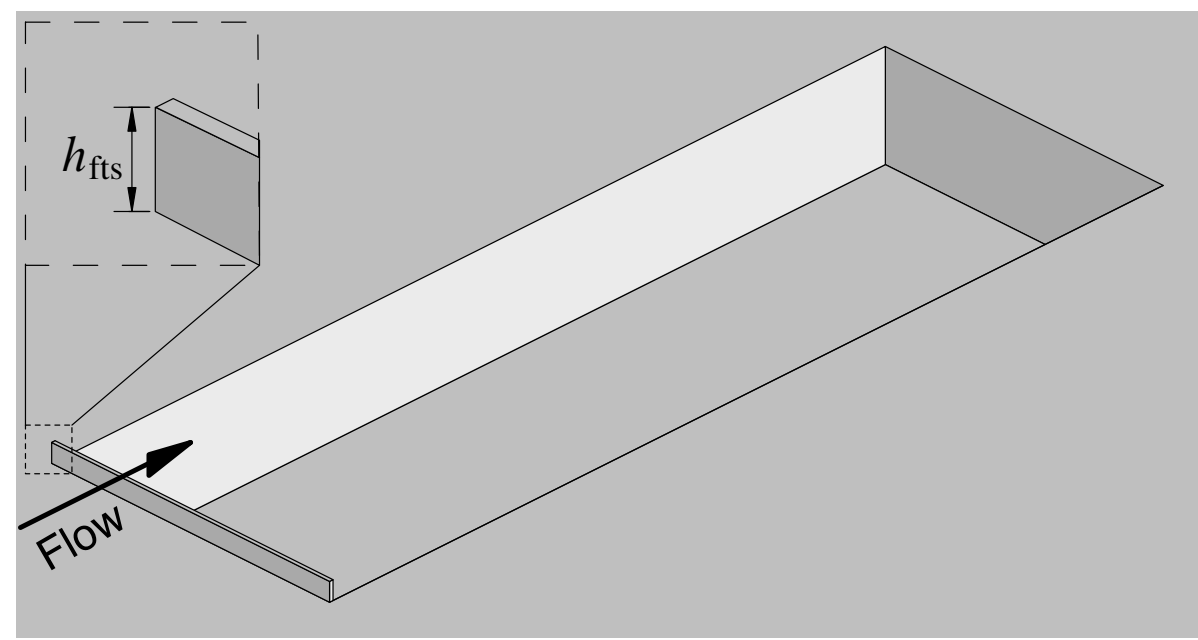

(a) Flat-top spoiler (FTS).

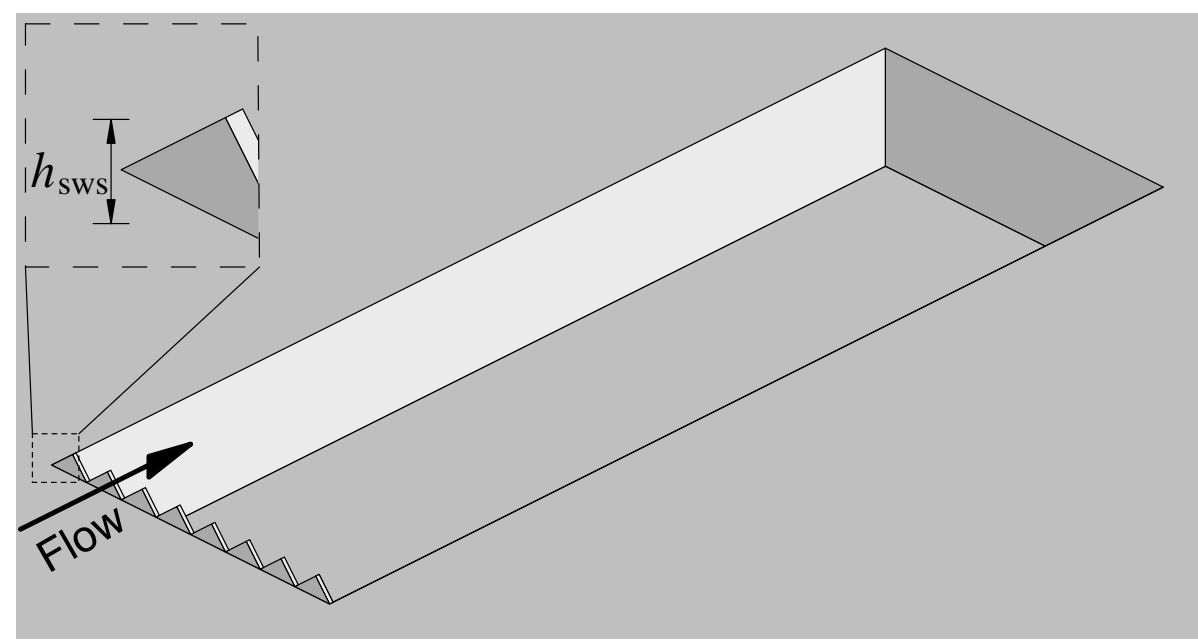

(b) Sawtooth spoiler (SWS).

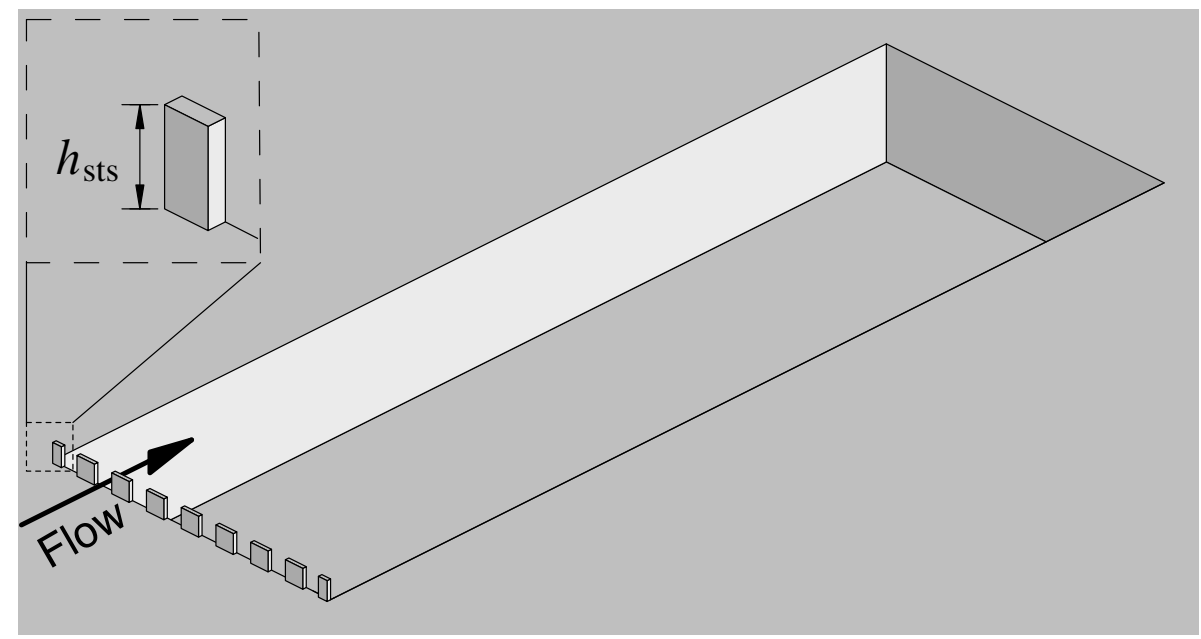

(c) Square-tooth spoiler (STS).

Figure 5: Passive control method geometries. 


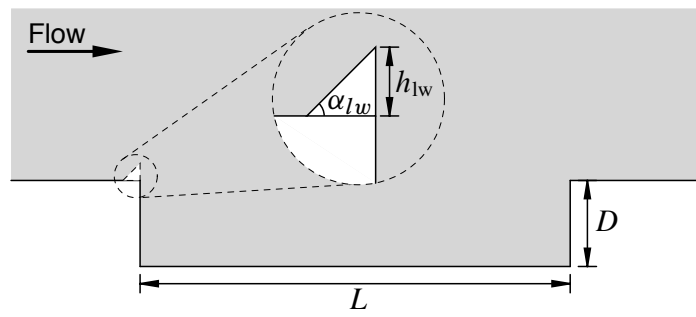

(d) Leading-edge wedge (LW).

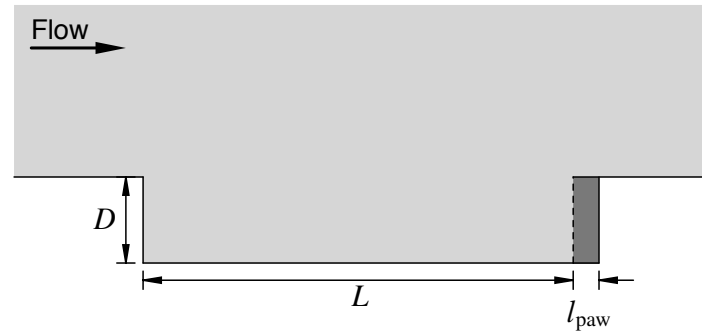

(f) Porous aft wall (PAW).

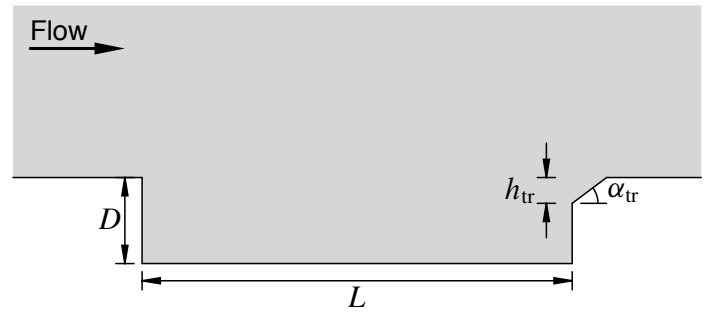

(h) Trailing-edge ramp (TR).

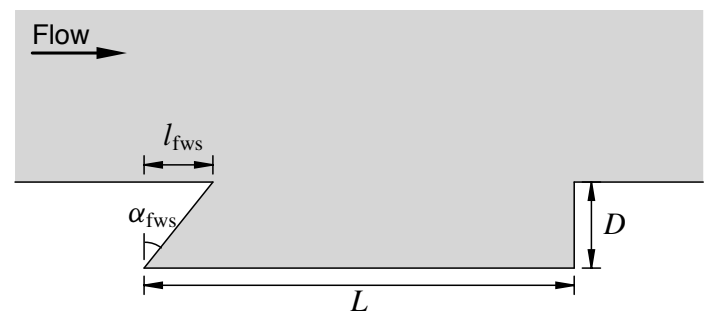

(j) Front-wall slant (FWS).

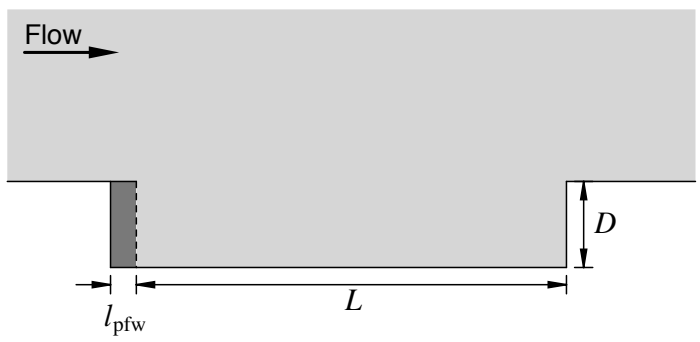

(e) Porous front wall (PFW).

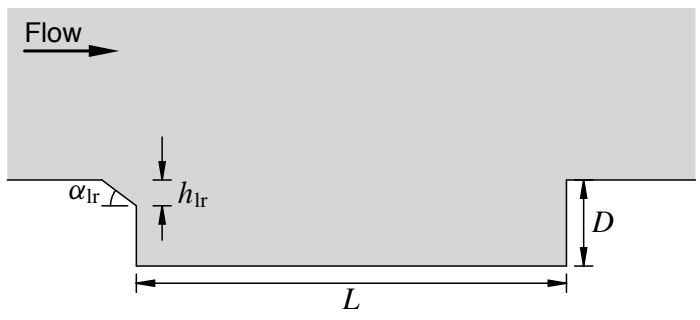

(g) Leading-edge ramp (LR).

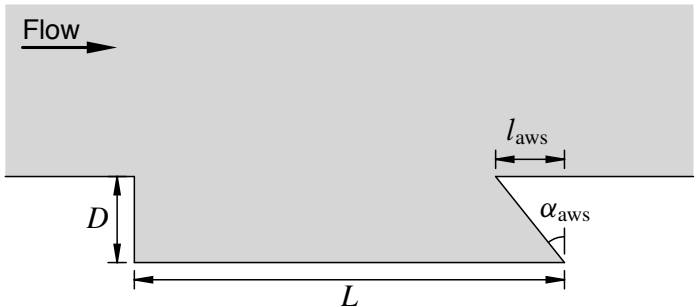

(i) Aft-wall slant (AWS).

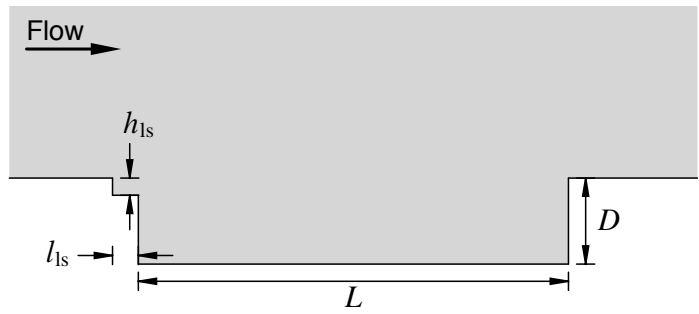

(k) Leading-edge step (LS).

Figure 5: Passive control method geometries (cont.). 


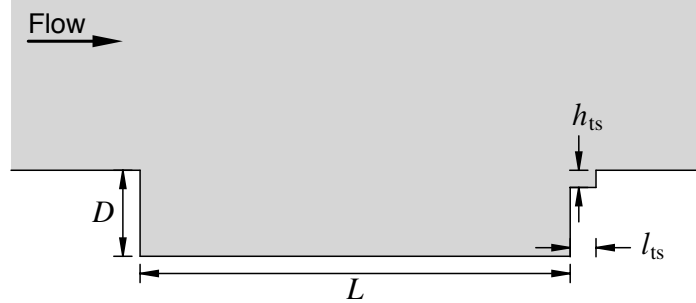

(I) Trailing-edge step (TS).

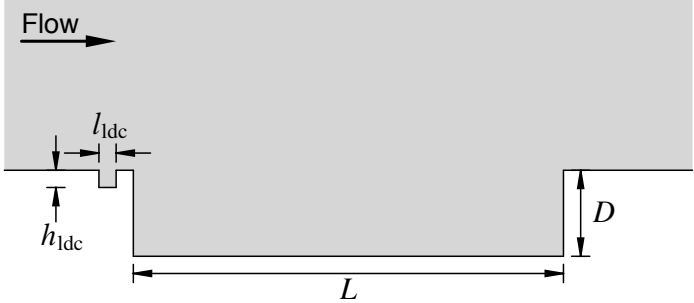

(m) Leading-edge deep cavity (LDC).

Figure 5: Passive control method geometries (cont.). 


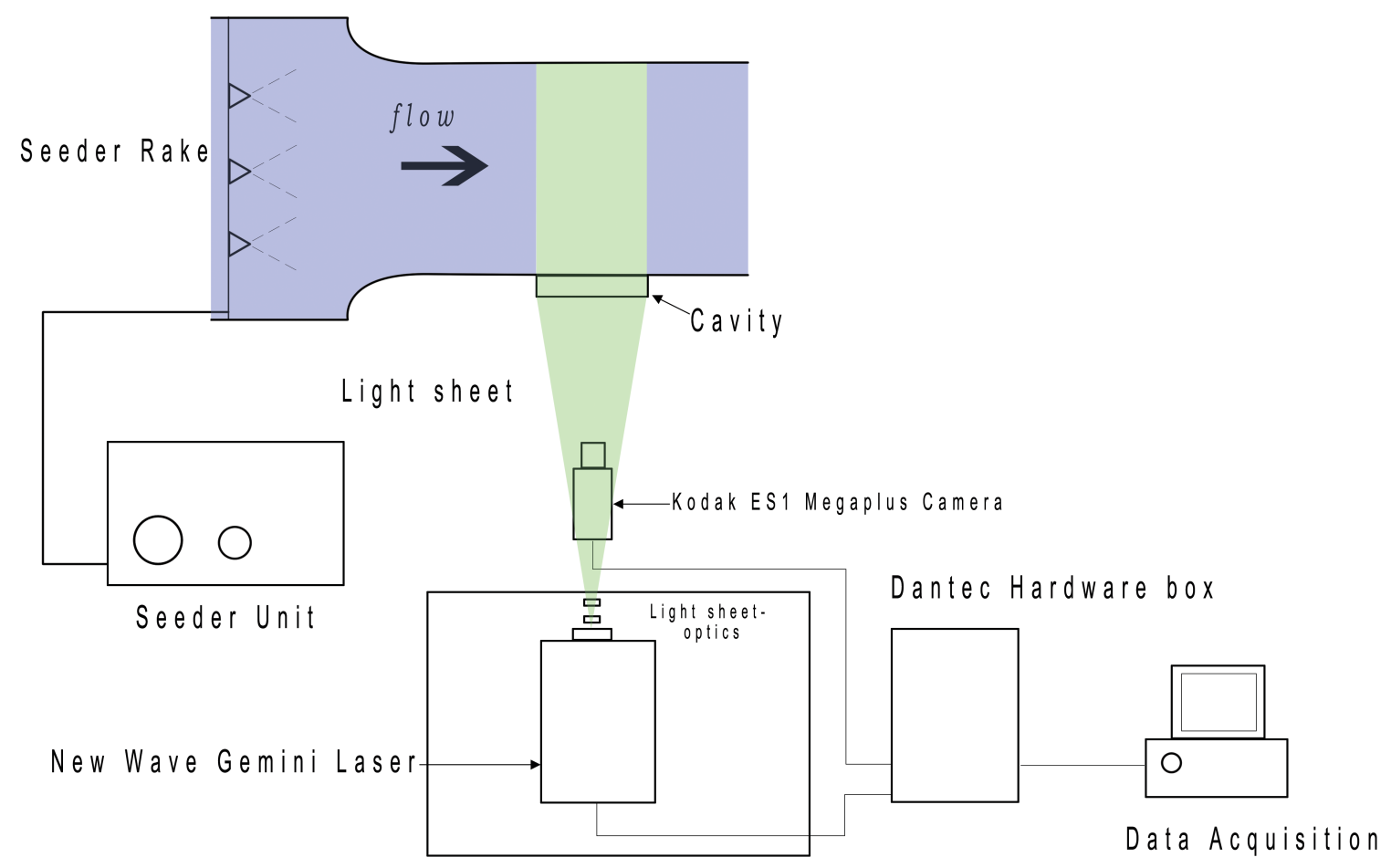

(a) Top view.

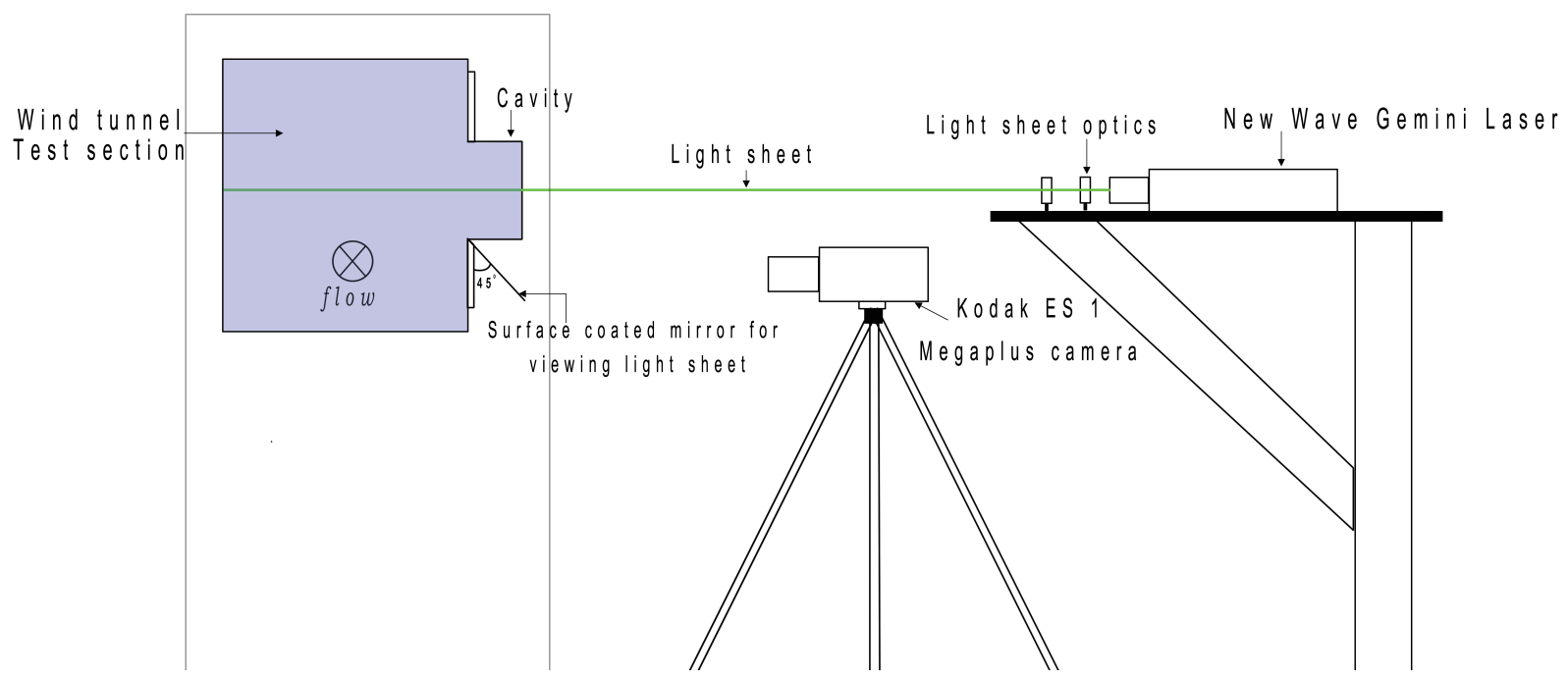

(b) Side view.

Figure 6: Schematic of the PIV setup. 


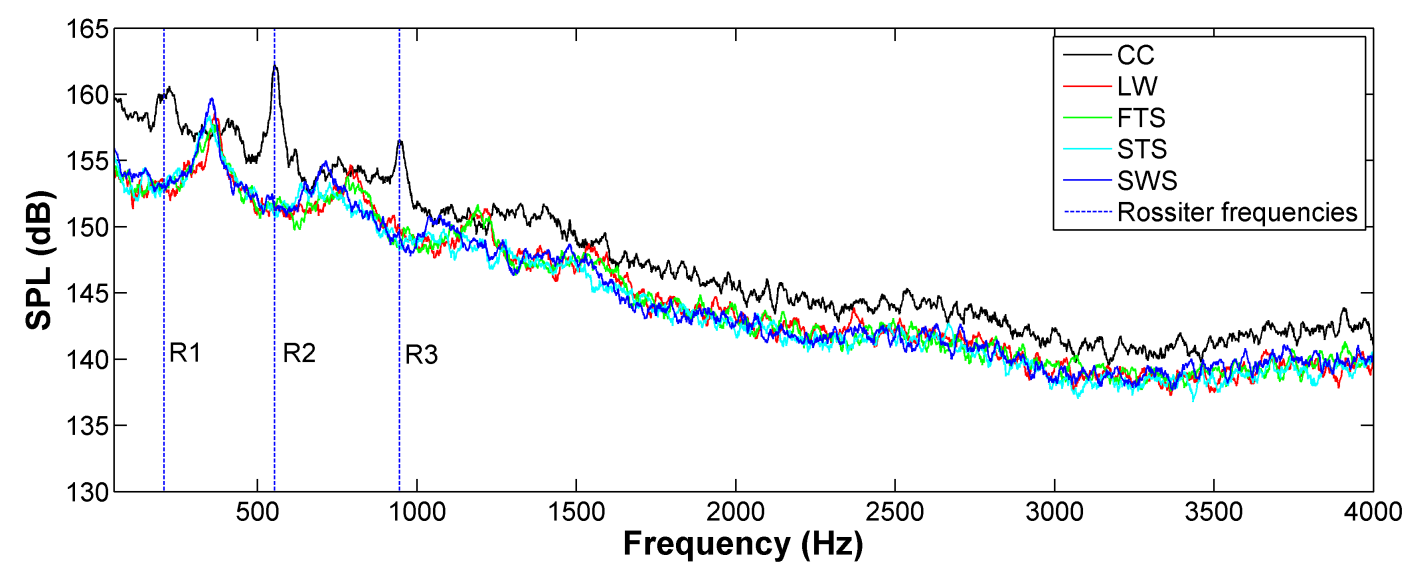

Figure 7: Effect of leading edge spoilers on the sound pressure level inside the cavity $\left(M_{\infty}=\right.$ $0.71, x / L=0.9)$.

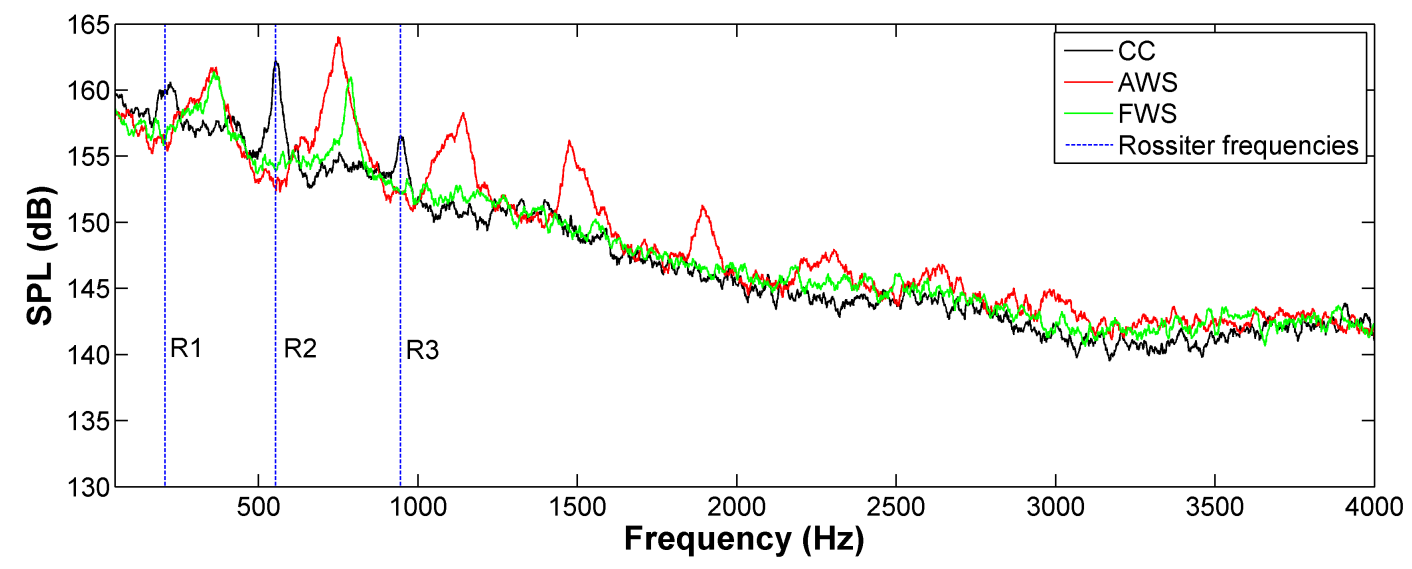

Figure 8: Effect of slant walls on the sound pressure level inside the cavity $\left(M_{\infty}=0.71, x / L=\right.$ 0.9). 


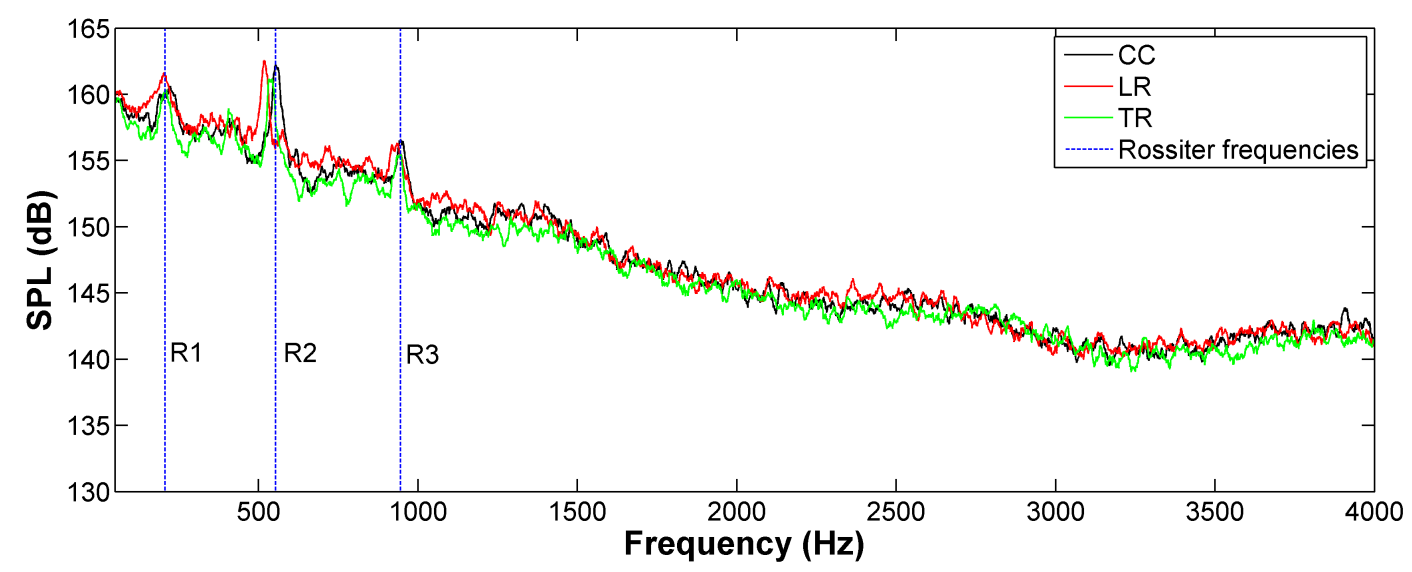

Figure 9: Effect of ramps on power sound pressure level inside the cavity $\left(M_{\infty}=0.71, x / L=\right.$ $0.9)$.

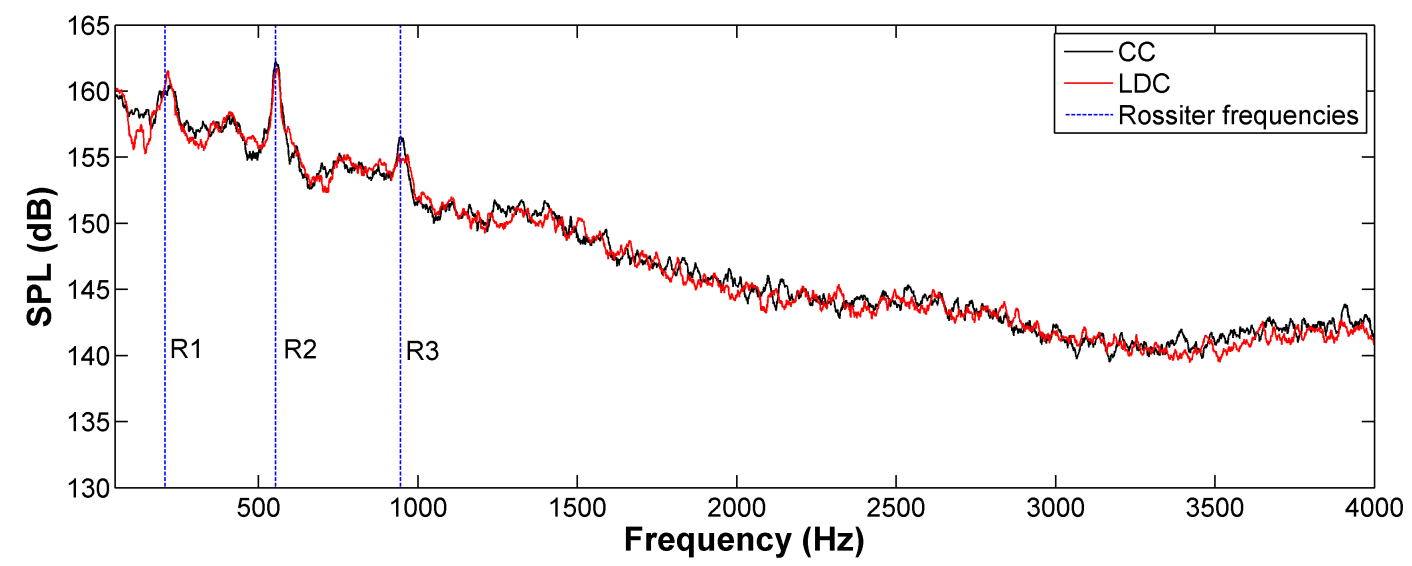

Figure 10: Effect of a leading-edge deep cavity (LDC) on the sound pressure level inside the cavity $\left(M_{\infty}=0.71, x / L=0.9\right)$. 


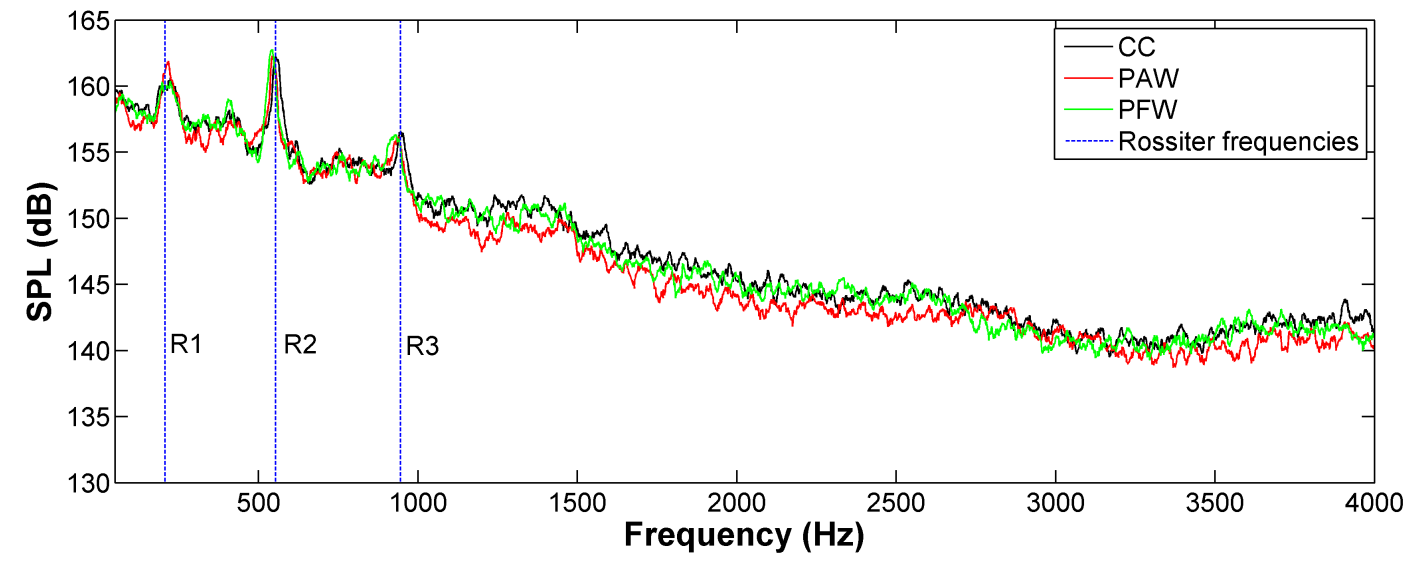

Figure 11: Effect of porous walls on the sound pressure level inside the cavity $\left(M_{\infty}=0.71, x / L=\right.$ 0.9 ).

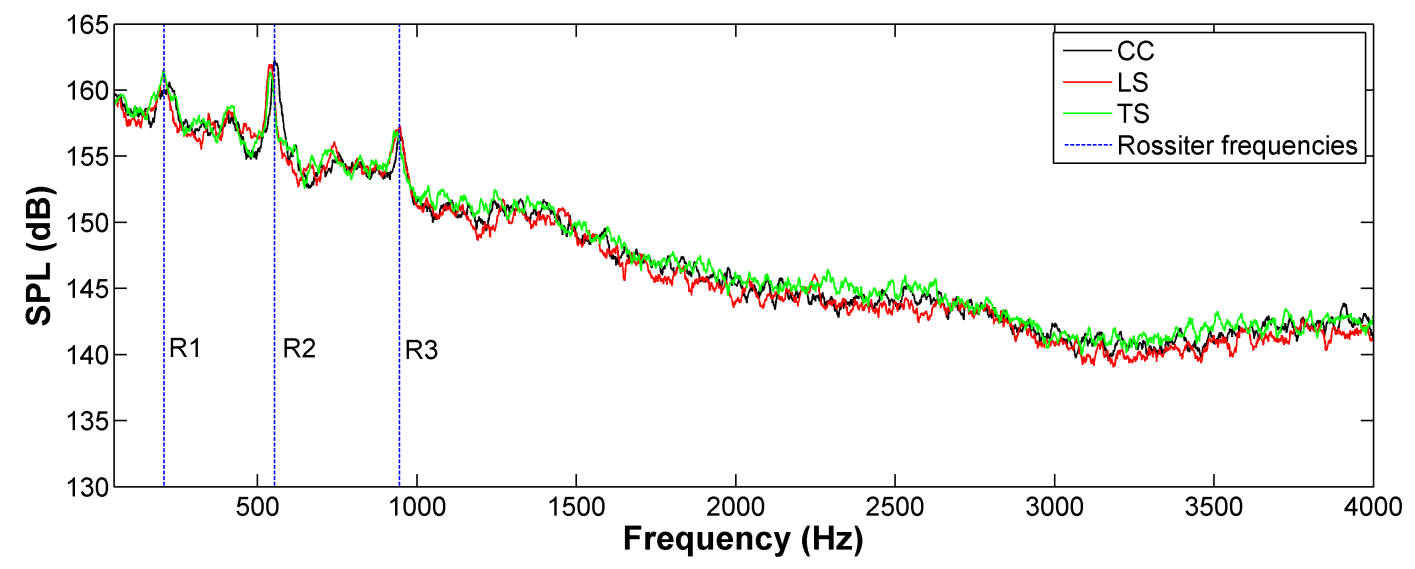

Figure 12: Effect of steps on the sound pressure level inside the cavity $\left(M_{\infty}=0.71, x / L=0.9\right)$. 


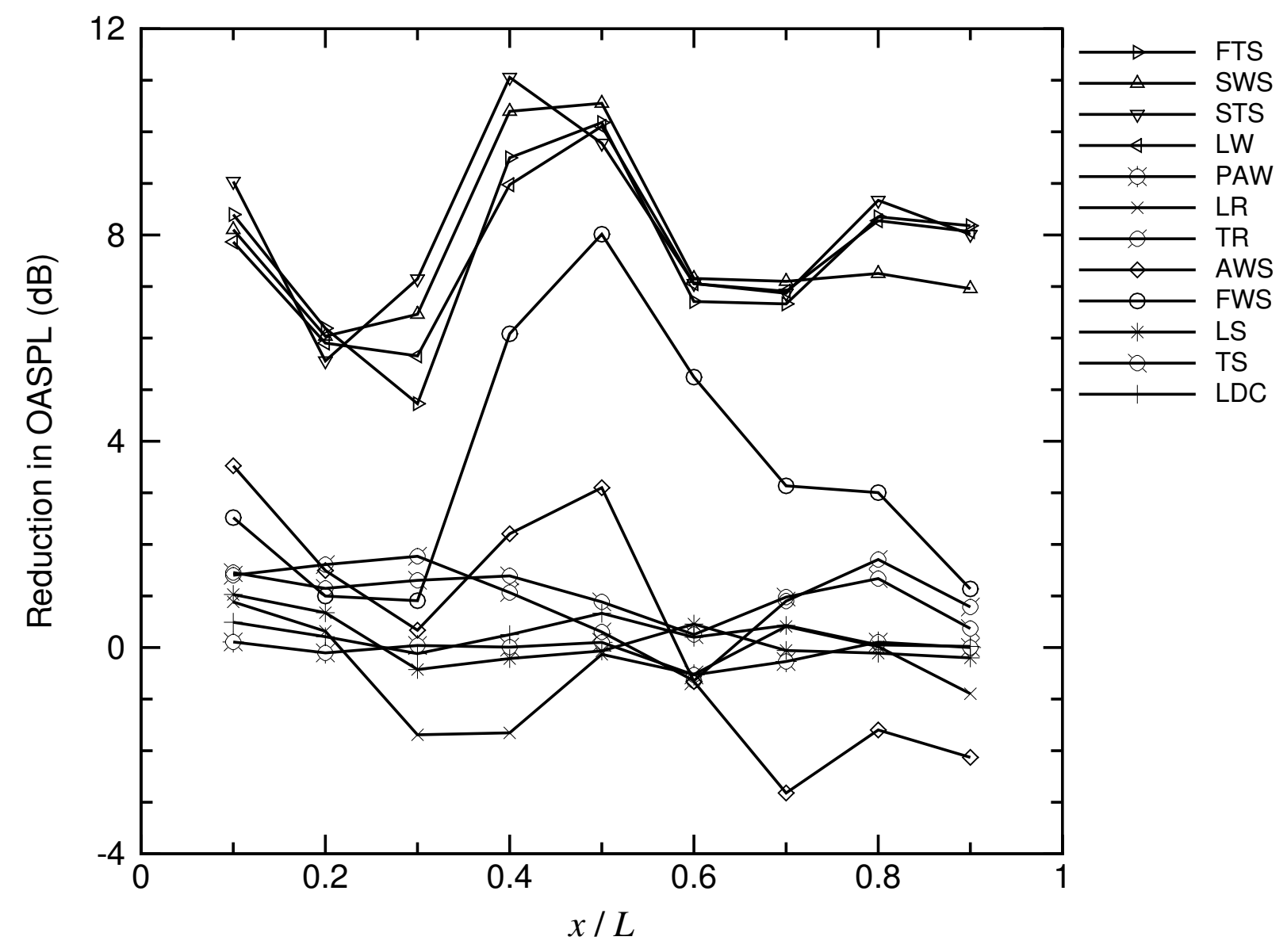

Figure 13: Effect of the different passive control devices on centreline OASPL $\left(M_{\infty}=0.71\right)$. 


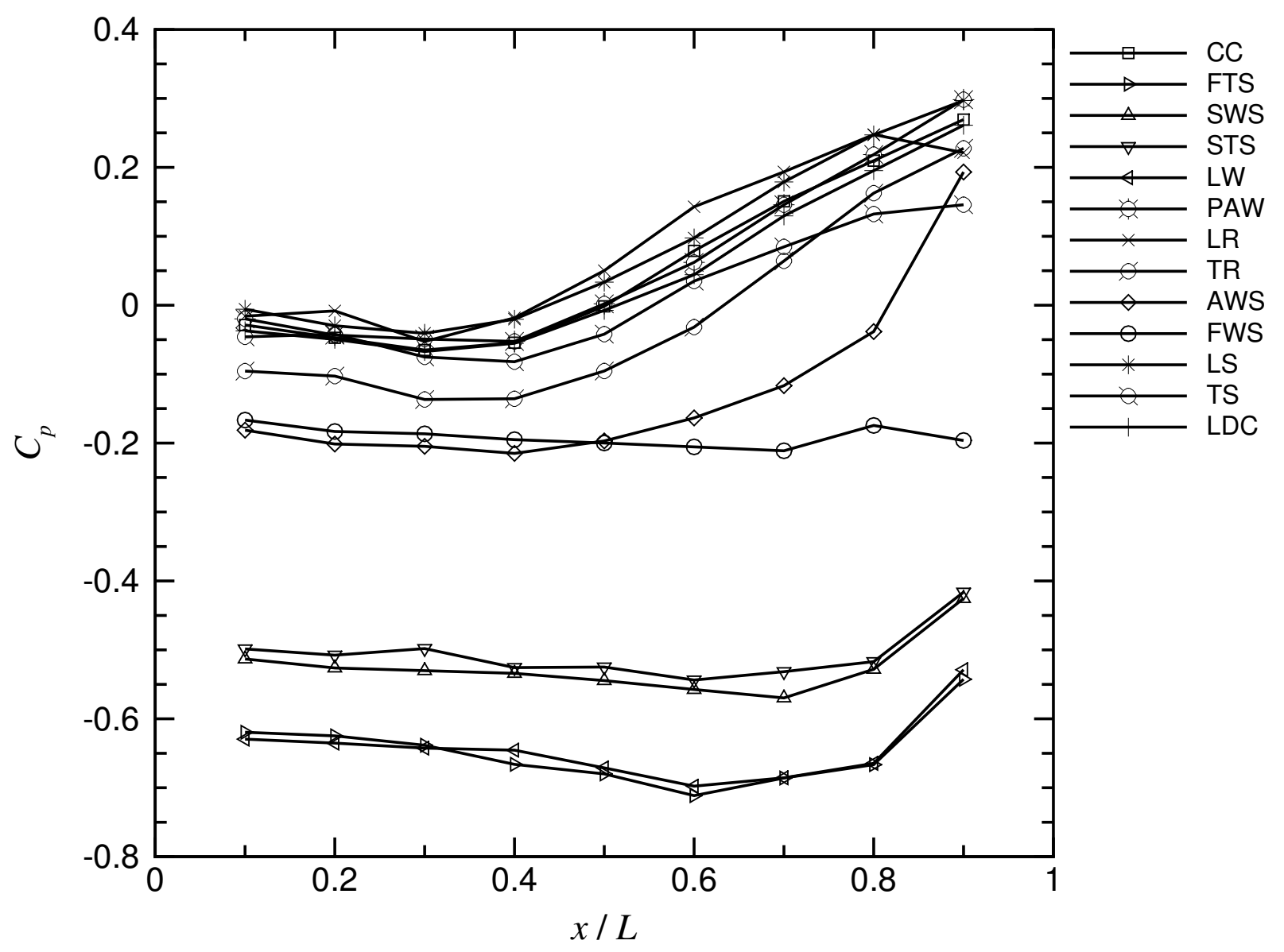

Figure 14: Effect of the different passive control devices on centreline mean pressure distribution $\left(M_{\infty}=0.71\right)$. 

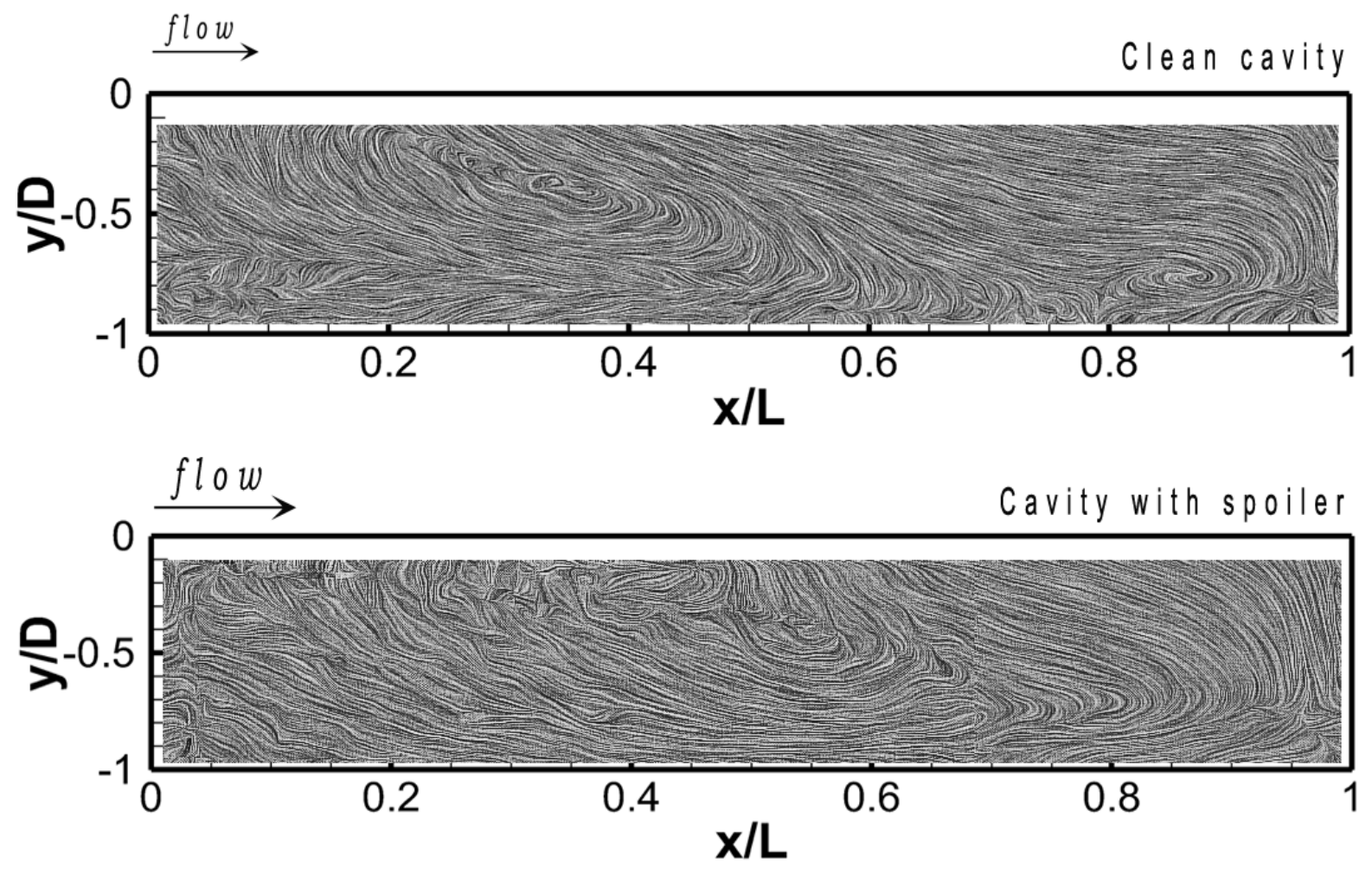

Figure 15: Line integral convolution images showing the effect of the sawtooth spoiler on the cavity flow $\left(z=0, M_{\infty}=0.71\right)$. 


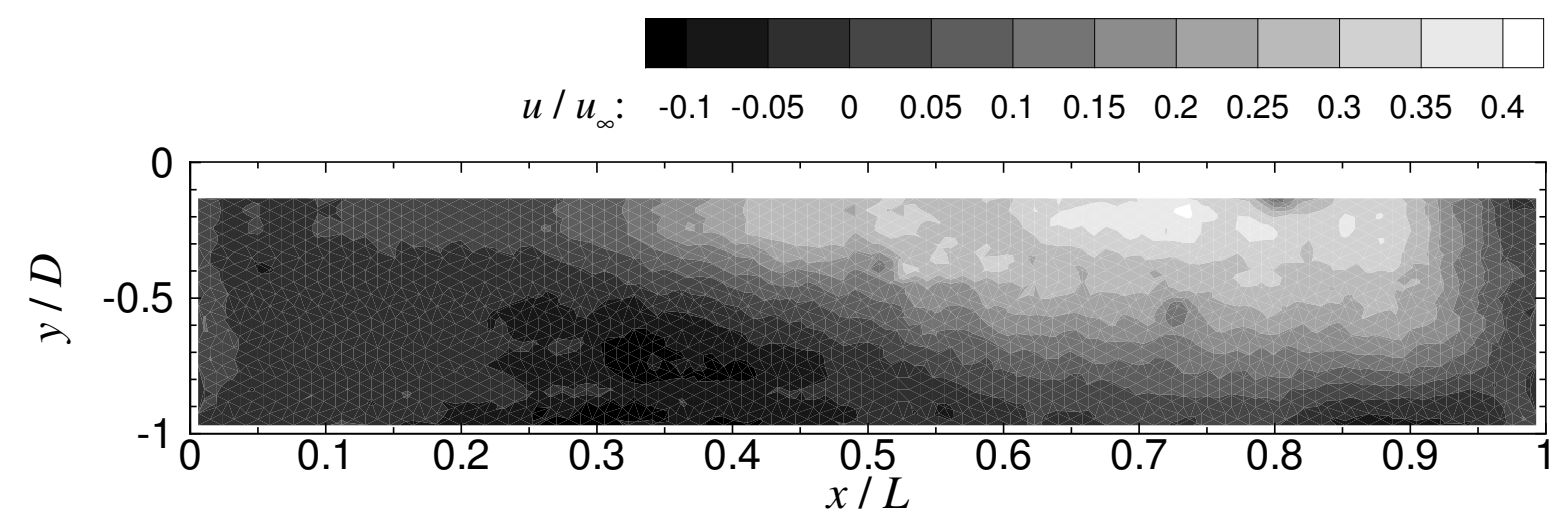

(a) Clean cavity.

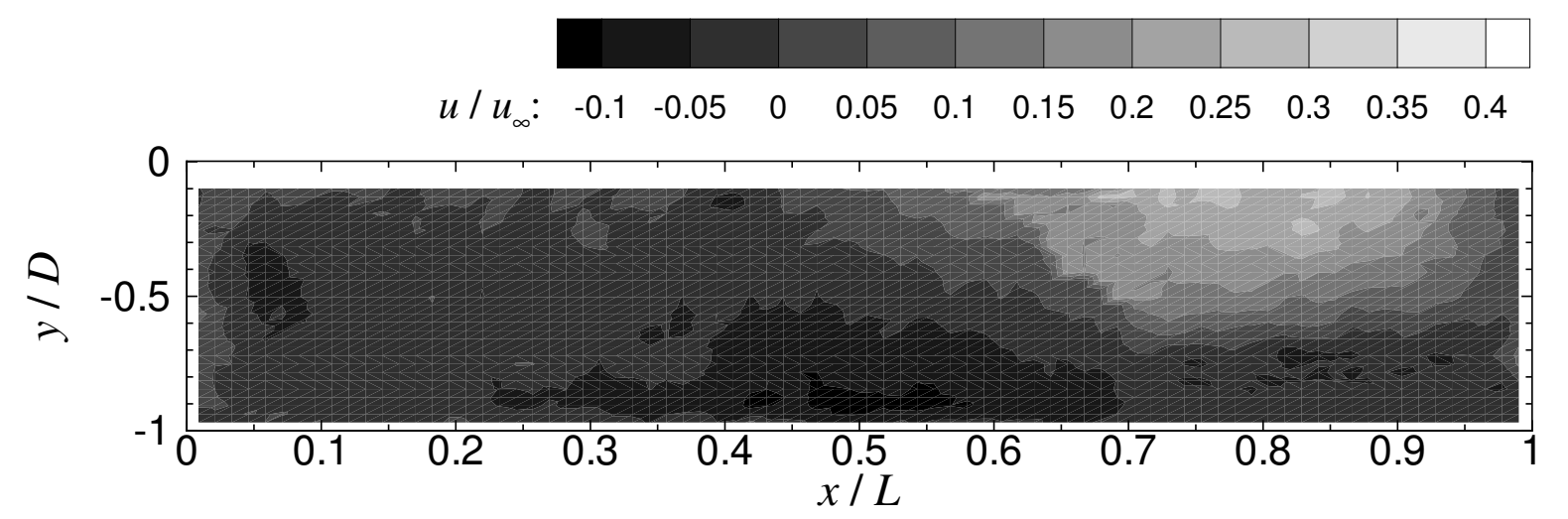

(b) Cavity with sawtooth spoilers.

Figure 16: PIV-derived contours of normalised streamwise velocity; freestream flow is from left to right $\left(z=0, M_{\infty}=0.71\right)$. 


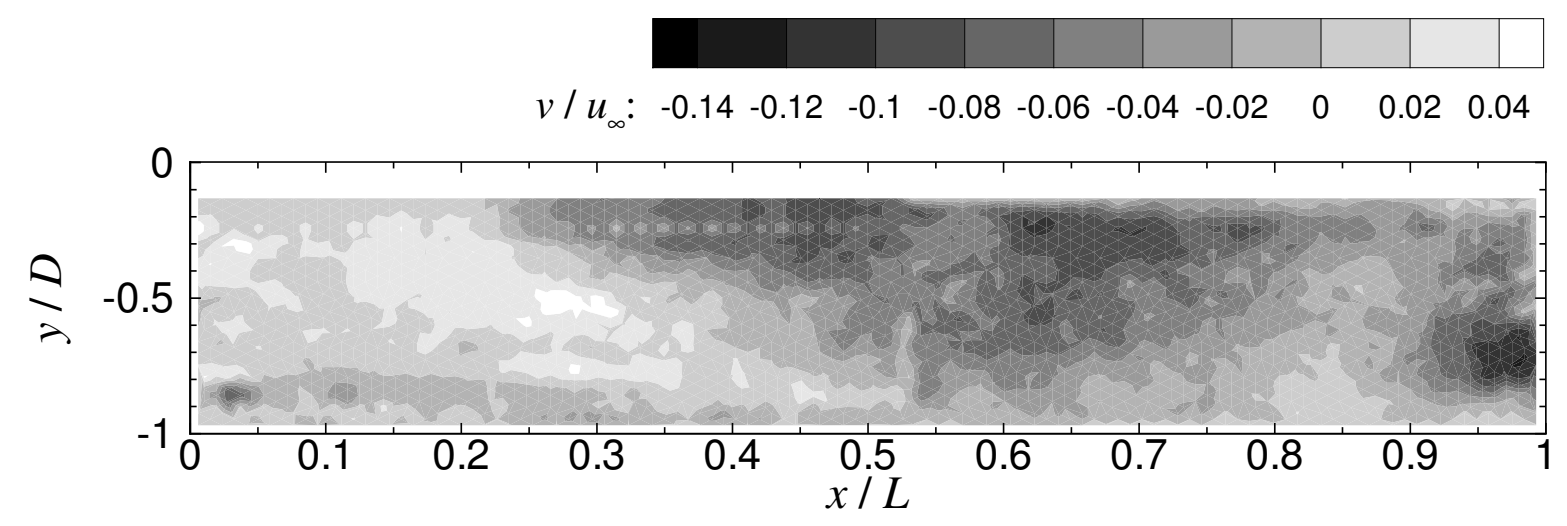

(a) Clean cavity.

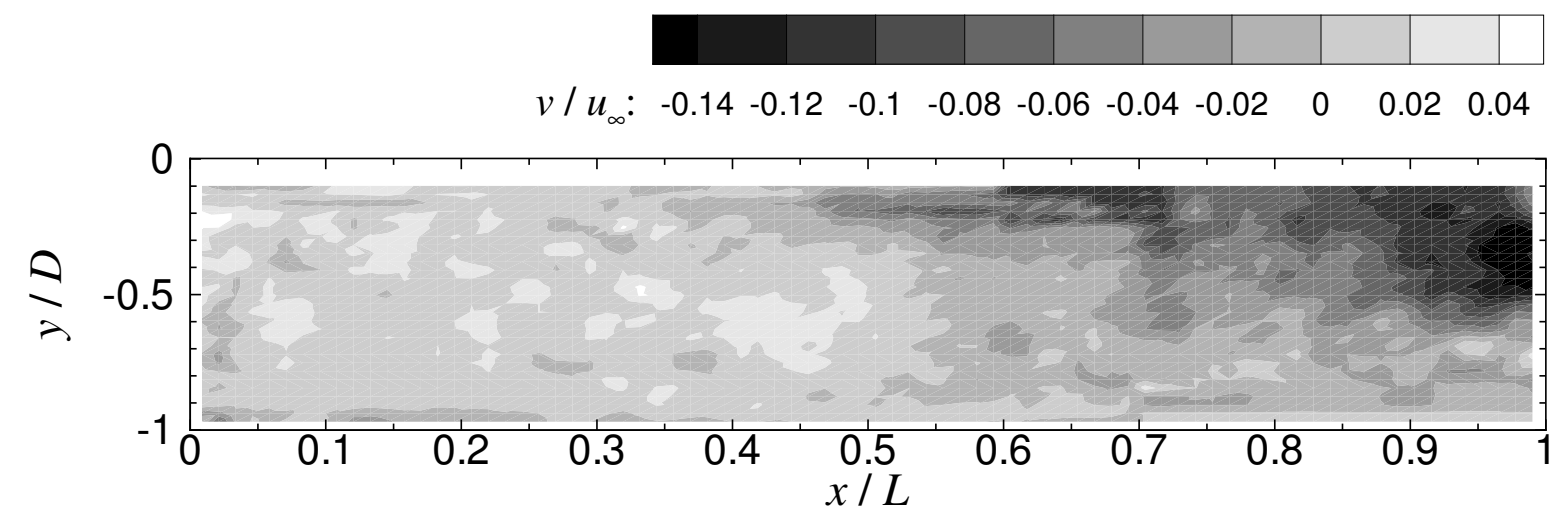

(b) Cavity with sawtooth spoilers.

Figure 17: PIV-derived contours of normalised vertical velocity; freestream flow is from left to right $\left(z=0, M_{\infty}=0.71\right)$. 